\title{
Helical CT of abdominal aortic aneurysms prior to endovascular stent graft repair
}

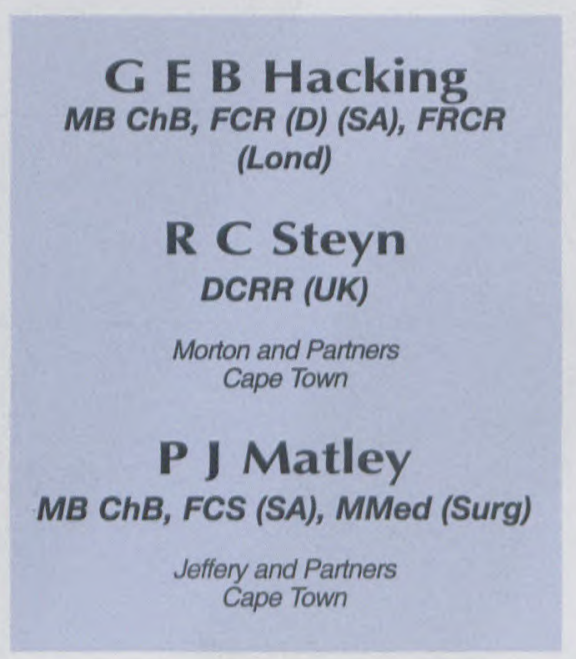

\section{Introduction}

Conventional abdominal aortic aneurysm (AAA) repair carries significant mortality and morbidity risk. Endovascular repair using a stent graft introduced through small femoral cut-downs is an alternative treatment that is minimally invasive and associated with a greatly reduced hospital stay, no requirement for postoperative intensive care and a rapid return to normal physical activity. It is therefore not surprising that this relatively noninvasive percutaneous technique of placing an endovascular stent graft (ESG) for repair of AAAs has become the treatment preferred by many vascular surgeons for suitable AAAs.
Endovascular repair is currently limited by its considerable cost, the lack of adequate long-term follow-up information proving its durability and the fact that currently only approximately $50 \%$ of aneurysms are anatomically suitable for ESG repair. Case selection depends on careful evaluation of the position of the renal arteries, the 'neck' of the aneurysm, the presence of patent lumbar or inferior mesenteric arteries and the state of the iliac arteries. Vascular surgeons rely on radiologists to provide highquality imaging in order to make this assessment. Although some units continue to employ conventional aortography in addition to helical CT imaging, most vascular surgeons are currently planning these procedures using $\mathrm{CT}$ alone.

\section{Aims of CT examination}

The aim of imaging prior to ESG placement is to: (i) determine if the AAA is suitable for repair with ESG; (ii) measure the dimensions of the abdominal aorta, the aneurysm and the iliac arteries in order to size the ESG accurately; and (iii) assess and document the relevant vascular anatomy, including branch vessel vari- ants, degree of tortuosity and extent of mural calcification and thrombus.

\section{Technique $e^{1-4}$}

A localising pre-contrast helical CT study is performed from the diaphragm to the symphysis pubis. The protocol is an $8 \mathrm{~mm}$ slice with a table feed of $16 \mathrm{~mm}$ (pitch of 2) and a reconstruction increment of $10 \mathrm{~mm}$. This pre-contrast study facilitates accurate planning of the scan range for the contrast enhanced helical CT angiogram. The range must begin just superior to the origin of the coeliac artery and end when a clear distinction can be made between the external and internal iliac arteries.

The scan protocol for the contrast enhanced helical CT angiogram is as follows: $3 \mathrm{~mm}$ slice thickness, pitch of 2 , reconstruction increment $1.5 \mathrm{~mm}$, craniocaudal scan direction, $150 \mathrm{ml}$ of low osmolar contrast IV at $4.5 \mathrm{ml}$ per second

If possible, a bolus-tracking programme should be used and the scan triggered at 90 Hounsfield units $(\mathrm{HU})$. If one is not available, then use a pre-programmed delay of approximately 20 - 25 seconds.

The scan can then be loaded into a $3 \mathrm{D}$ post processing programme and the aorta (from just proximal to the coeliac axis) and the iliac arteries (down to just distal to the common iliac arteries' bifurcations) can be edited out from the rest of the abdominal and pelvic structures. The following anatomical landmarks should be included in the edited 3D data set: (i) the origin of the coeliac axis; (ii) the origin of the SMA; (iii) the origins of the renal arteries and accessory renal arteries; and (iv) the thrombus, and any calcification 


\section{ORIGINAL ARTICLE}

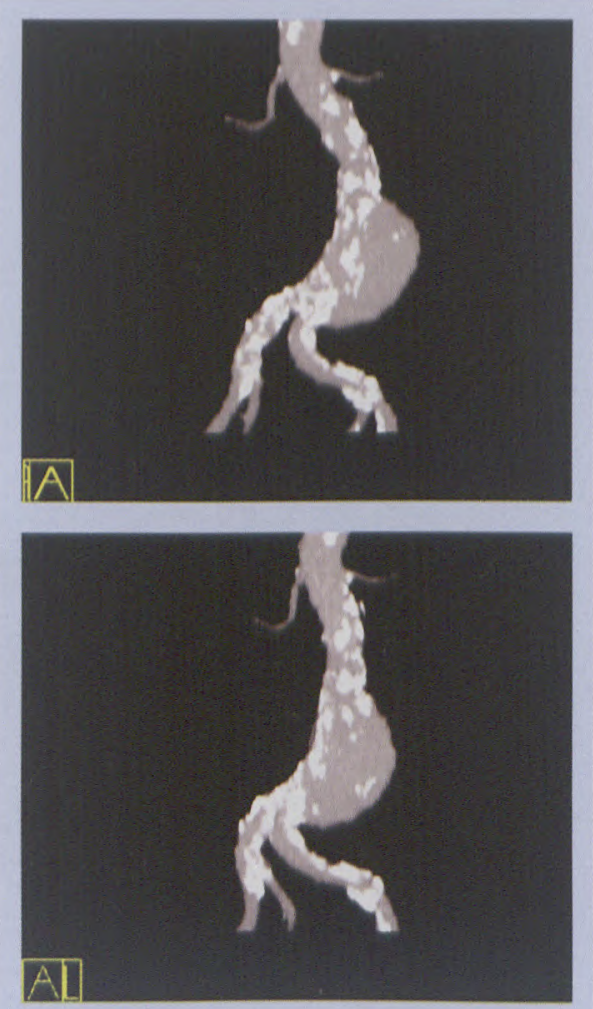

Figs. 1 and 2. Examples of reconstructed MIP images showing abdominal aorta with an infrarenal aneurysm, left and right renal arteries and left and right iliac artery bifurcations. Note the favourable long infrarenal neck and the moderate to severe mural calcification.

around the aorta, must be included as well as the lumen - this is to provide a guide to the extent of the aneurysm in post processing.

A range of high-resolution MIP (maximum intensity projection) images can be taken from these edited images. We usually reconstruct 12 images through $180^{\circ}$ about the Z-axis, two of which are demonstrated in Figs 1 and 2.

\section{Report}

The following questions should be answered by the report:

1. Is the AAA supra- or infrarenal? Suprarenal AAAs are not suitable for routine ESG placement.

2. What is the length of the proximal neck of the AAA? This is the dis- tance from the origin of the most inferior renal artery to the origin of the AAA. If this is less than $10 \mathrm{~mm}$ then the AAA is probably not suitable for ESG placement.

3. Is the neck diameter uniform rather than 'coned' or 'flared'?

4. Is there thrombus present in the neck?

5. Are there accessory renal arteries and what are their positions relative to the AAA?

6. Is the inferior mesenteric artery patent and are there large patent lumbar arteries arising from the aneurysm?

7. What is the true length of each common iliac artery?

8. Are the iliac arteries aneurysmal, stenosed, excessively tortuous or calcified?

The report should obviously include any signs of AAA complications and any relevant incidental abdominal or pelvic abnormalities. If there are no anatomical contraindications to ESG placement then the measurement sheet should be completed and included with the report.

\section{Measurements}

In order to achieve a successful repair of AAA with ESG placement, the correct size ESG must be ordered from the manufacturer. A measurement sheet, supplied by the manufacturer, should be completed (Fig. 3). Not only should the length and diameter of the arteries and AAA be documented, but also an assessment of arterial tortuosity, mural thrombus formation and wall calcification should be recorded.

We use routine axial cross-section-

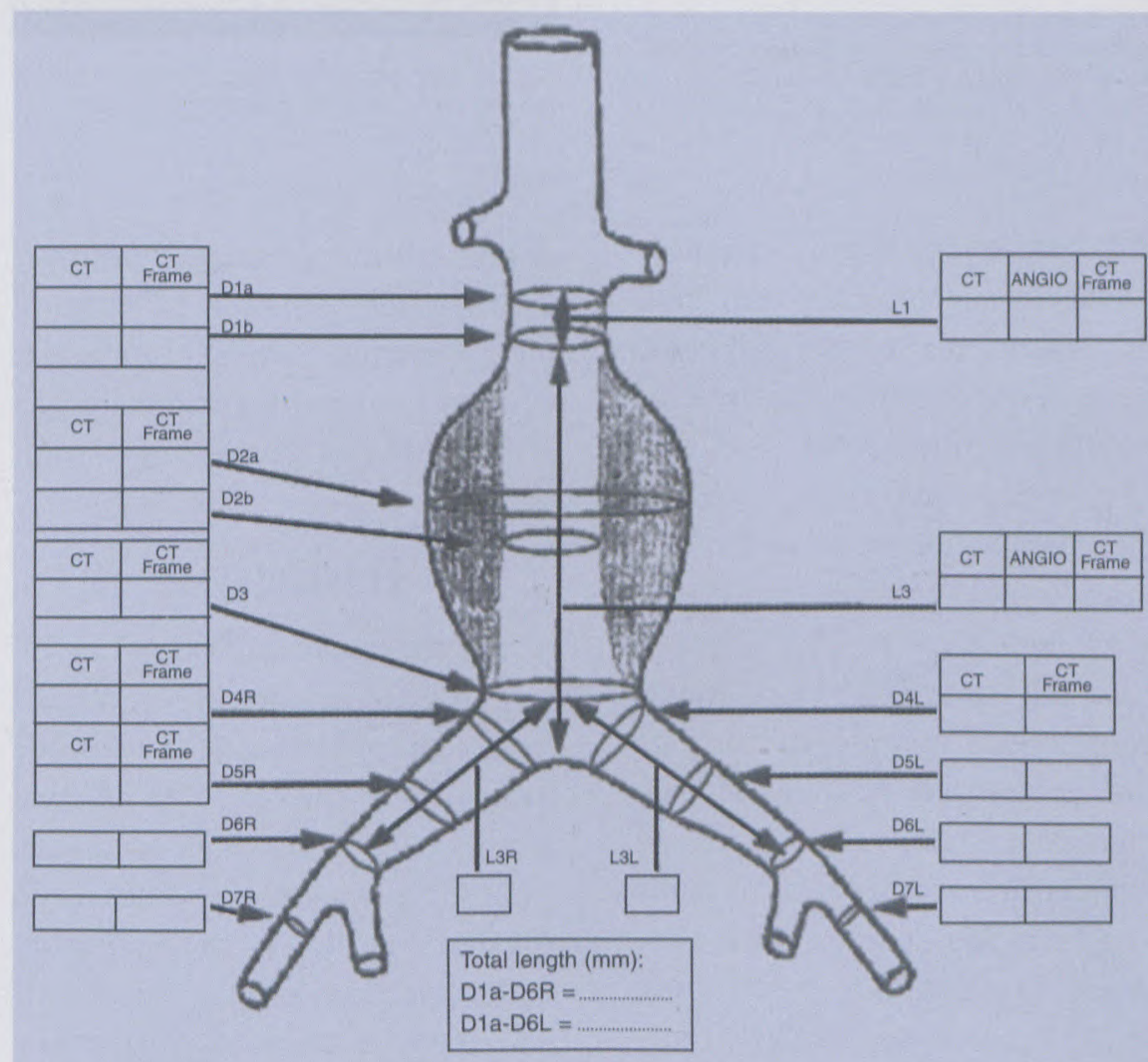

Fig. 3. Worksheet demonstrating measurements required. 


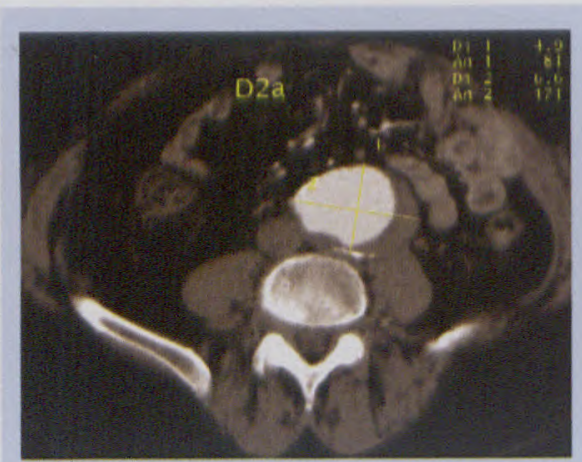

Fig. 4. Axial CT image showing the cross-sectional measurements of the maximum diameter of the wall of the aneurysm (D2a).

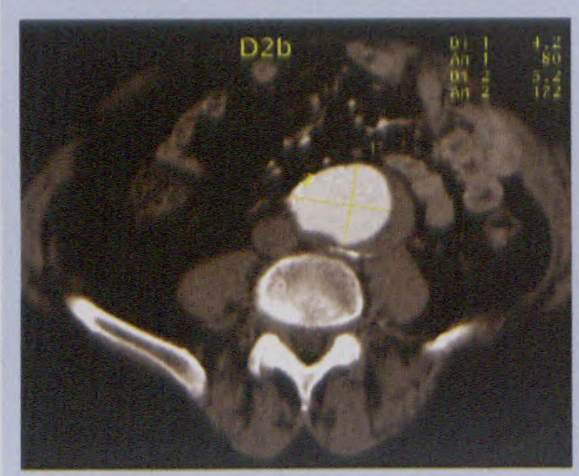

Fig. 5. Axial CT image showing the cross-sectional measurements of the maximum diameter of the lumen of the aneurysm (D2b).

al CT images (Figs 4 and 5) to measure diameters of vessels with long axes perpendicular to the axial plane (the usual orientation of the abdominal aorta and most AAAs).

However, for vessels with an oblique orientation we prefer the MIP images to measure diameters (Figs. 6 and 7). For similar reasons we prefer to use MIP images for vessel length measurements because calculations based on the table positions of axial images do not take vessel obliquity or tortuosity into account, which results in length underestimation due to geometrical foreshortening. When using MIP 3D reconstructed images to measure diameters and lengths it is important to view these images on a work

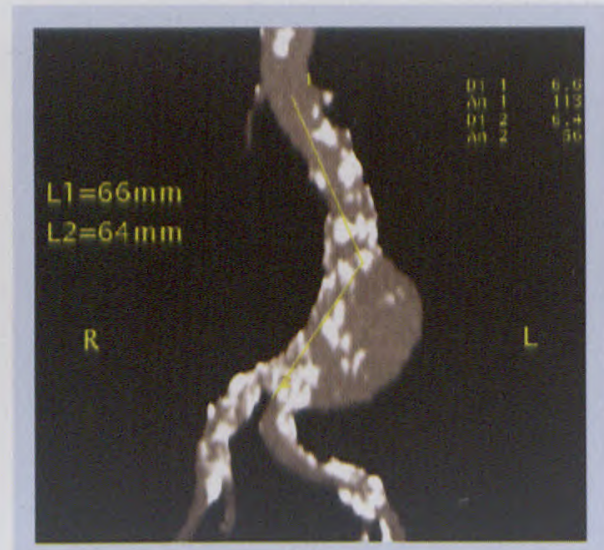

Fig. 6. MIP image showing measurement of length of aneurysm neck (L1) and length of aneurysm (L2).

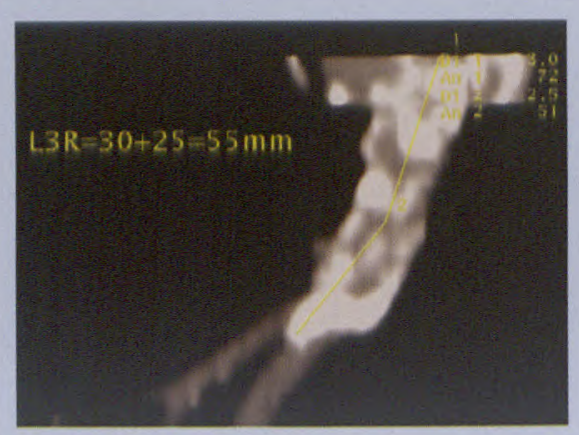

Fig. 7. MIP image showing measurement of length of right common iliac artery (L3R).

station in order to rotate the images appropriately so that geometrical foreshortening (which can occur whenever a three-dimensional object is viewed in two dimensions) is minimised.

\section{Imaging}

For the hard copy, it is not necessary to give the surgeon every axial image, except through the following structures: $(i)$ the origins of all renal arteries; (ii) the neck of the aneurysm; (iii) the bifurcation of the aorta; and (iv) the bifurcation of the iliac arteries.

A selection of the remaining images can be included, along with a film of the measurements and the 3D MIP images (Figs 8-11).

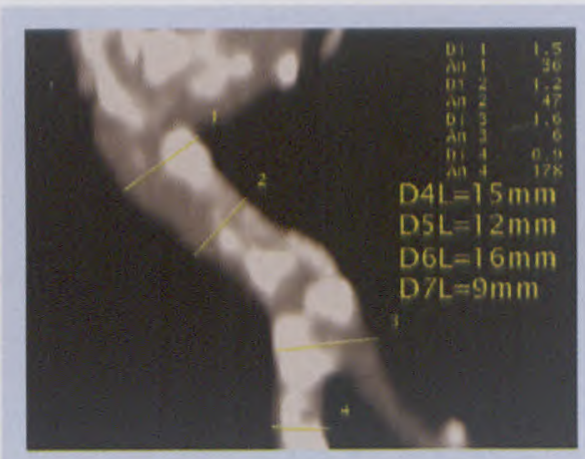

Fig. 8. MIP image showing measurements of diameters of left common iliac and left external iliac arteries (D4L, D5L, D6L, D7L).

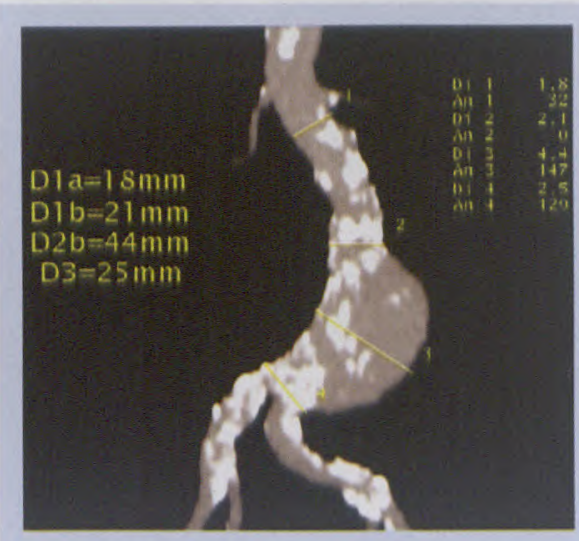

Fig. 9. MIP image showing diameter measurements of proximal (D1a) and distal (D1b) neck, lumen of aneurysm (D2b) and distal aorta (D3).

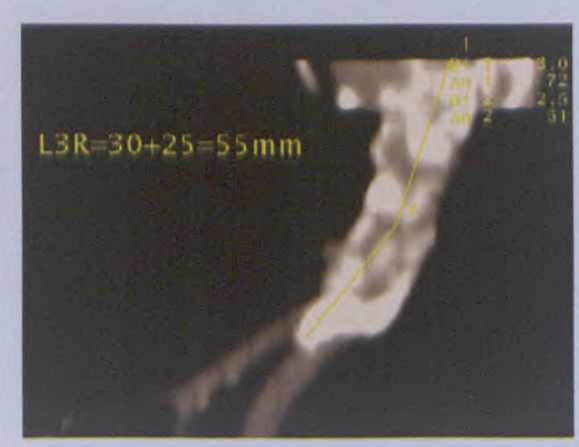

Fig. 10. MIP image showing length measurement of right common iliac artery (L3R).

\section{Surgical perspective}

The important criteria that disqualify an individual patient from any consideration for endovascular repair relate to a short or excessively angulat- 


\section{ORIGINAL ARTICLE}

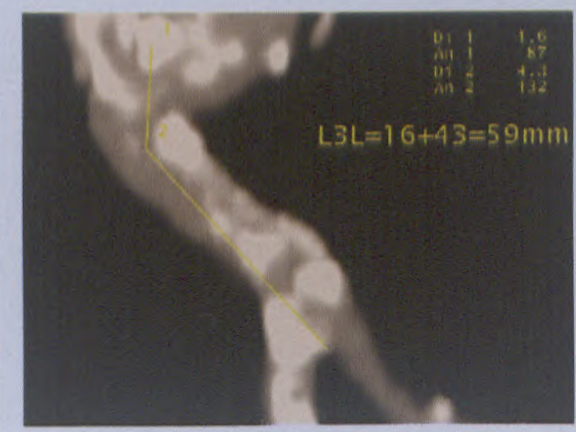

Fig. 11. MIP image showing length measurement of left common iliac artery (L3L).

ed infra-renal neck, the presence of thrombus in the neck, aneurysms of the common iliac arteries that involve the iliac bifurcation bilaterally and iliac arteries that are narrow in calibre or excessively calcified and tortuous. Once the surgeon is satisfied that none of these contraindications exist, precise measurements of diameter and length are essential at every level. Modular stent graft systems demand precise sizing so that each component fits the patient's anatomy. Some devices such as the Talent and Lifepath are individually constructed on a per-patient basis according to the basic CT images that are provided to the company in the USA, usually through electronic data transfer. Several systems such as the Zenith and AneuRx involve ordering from a limited range of standard components according to the patient's anatomy. The stent graft is then constructed within the aneurysm by deploying the various components into one another. The Endologix device represents a new generation of non-modular endovascular stent grafts with a revolutionary 'one size fits all' philosophy. This, however, does not eliminate the need for precise measurement, as these systems are still only able to accommodate a limited range of aortic and iliac sizes. Vascular surgeons spend a great deal of time planning each individual procedure and ordering each individual stent graft. Errors at this stage can mean the difference between a successful endovascular procedure and a complex emergency conversion to open surgery. Although some errors can be compensated for using various stent-graft extensions, these increase the cost of the procedure considerably.

\section{Conclusion}

A high quality helical CT angiographic study is usually capable of providing all the imaging information necessary to assess whether the AAA in question is technically suitable for successful ESG placement. It also allows for the relevant vascular measurements to be acquired in order to size the ESG accurately. In the vast majority of cases no further imaging is necessary. In order to acquire relevant and accurate imaging information, the following requirements are necessary: (i) a high quality helical CT scanner capable of $3 \mathrm{~mm}$ slice helical 3D data set acquisition over a length of at least $30 \mathrm{~cm}$; (ii) a workstation with software appropriate for 3D MIP reconstructions and distance measurements; (iii) a dedicated, specialist CT radiographer with specific training and experience in CT angiography and 3D image data set editing and reconstructions; and (iv) good communication between the vascular surgeon and radiologist to ensure that all the relevant imaging information is accurately acquired and reported.

With the right equipment, technique and team approach, this technically demanding and time-consuming investigation can become a 'routine' non-invasive radiological investigation, providing all the necessary imaging information necessary prior to ESG placement.

\section{References}

1. Maatsch K, Knapheide C. Scan Protocols Somatom Plus 4. Erlangen: Siemens Aktiengesellschaff, 1997.

2. Thurnher MM, Winkelbauer FW, Kretschme G, Polterauer P, Lamer J. Evaluation of abdominal aortic aneurysm for stent-graft placement: comparison of gadolinium-enhanced MR angiography versus helical CT angiography and digital subtraction angiography. Radiology 1997; 205: 341

3. Semba CP, Razavi MK, Kee ST, et al. Applications of spiral CT in endovascular aortic interventions. Seminars in Interventiona Radiology 1998; 15: 179.

4. Rydberg J, Kopecky KK, Johnson MS, Dalsing MC, Persohn SA. Stent grafting of abdomina aortic aneurysms: pre- and postoperative evaluation with multislice helical CT. J Comput Assist Tomogr 2001; 25: 580. 